\title{
Elastic Buckling Analysis of SSCF and SSSS Rectangular Thin Plates using the Single Finite Fourier Sine Integral Transform Method
}

\author{
Michael Ebie Onyia ${ }^{1}$, Eghosa. O. Rowland-Lato ${ }^{2}$ and Charles Chinwuba Ike ${ }^{3}$ \\ ${ }^{1}$ Department of Civil Engineering University of Nigeria, Nsukka, Enugu State, Nigeria. \\ ORCID: 0000-0002-0956-0077 \\ ${ }^{2}$ Department of Civil Engineering, University of Port Harcourt, Choba. Nigeria. \\ ORCID: 0000-0001-7289-0530 \\ ${ }^{3}$ Department of Civil Engineering, Enugu State University of Science and Technology, Enugu. \\ ORCID: 0000-0001-6952-0993
}

\begin{abstract}
The analysis of the stability problem of plate subjected to inplane compressive load is important due to the relatively poor capacity of plates in resisting compressive forces compared to tensile forces. It is also significant due to the nonlinear, sudden nature of buckling failures. This study presents the elastic buckling analysis of SSCF and SSSS rectangular thin plates using the single finite Fourier sine integral transform method. The considered plate problems are (i) rectangular thin plate simply supported on two opposite edges, clamped on one edge and free on the fourth edge; (ii) rectangular thin plate simply supported on all edges. The plates are subject to uniaxial uniform compressive loads on the two simply supported edges. The governing domain equation is a fourth order partial differential equation (PDE). The problem solved is a boundary value problem (BVP) since the domain PDE is subject to the boundary conditions at the four edges. The single finite sine transform adopted automatically satisfies the Dirichlet boundary conditions along the simply supported edges. The transform converts the BVP to an integral equation, which simplifies upon use of the linearity properties and integration by parts to a system of homogeneous ordinary differential equations (ODEs) in terms of the transform of the unknown buckling deflection. The general solution of the system of ODEs is obtained using trial function methods. Enforcement of boundary conditions along the $y=0$, and $y=b$ edges (for the SSCF and SSSS plates considered) results in a system of four sets of homogeneous equations in terms of the integration constants. The characteristic buckling equation in each case considered is found for nontrivial solutions as a transcendental equation, whose roots are used to obtain the buckling loads for various values of the aspect ratio and for any buckling modes. In each considered case, the obtained buckling equation is exact and identical with exact expressions previously obtained in the literature using other solution methods. The buckling loads obtained by the present method are validated by the observed agreement with results obtained by previous researchers who used other methods.
\end{abstract}

Keywords: single finite Fourier sine integral transform method, characteristic elastic buckling equation, critical elastic buckling load, elastic buckling load coefficient, elastic buckling problem.

\section{INTRODUCTION}

The analysis of stability problems of plate carrying in-plane compressive loads is important in structures due to the relatively poor capacity of plates in resisting compressive forces [ $1-7]$. It is also important due to the nonlinear, sudden nature of buckling. A good knowledge of buckling loads and the associated buckling mode shapes is fundamental to structural analysis and design for compressed plates; hence the need for development of effective analytical and numerical methods for solving the buckling problem.

The determination of buckling loads is thus an important theme in structural analysis. In-plane compressive loads can be uniformly or non-uniformly applied over the edges uniaxially or biaxially. The plate may be modelled as a thin or thick plate, depending on the thickness-width ratio, where the width is the least in-plane dimension.

The buckling problem is classified as elastic or inelastic. In elastic buckling problems, the critical buckling load is smaller than the elastic limit of the material otherwise the problem is called inelastic buckling.

Navier's investigation of stability problem of rectangular thin plates was one of the first studies on the subject of thin plate stability. Navier used the assumptions and hypotheses of the Kirchhoff thin plate theory to derive the stability equation of rectangular thin plates that included the twisting term.

Saint-Venant later presented a modification of the Navier's equation to include the axially applied edge loads and the shearing loads. Saint-Venant's modified differential equation provided the theoretical basis for the elastic stability of thin plates with various edge loads and edge support conditions.

Bryan used the total potential energy minimization principle to solve the elastic stability of a rectangular thin plate under uniaxial compressive load for Dirichlet boundary conditions. Bryan assumed the buckling shape function for the problem as a double Fourier sine series.

Timoshenko [6] also presented solutions to elastic stability problem of rectangular thin plates with simply supported edges under uniaxial compressive loads by assuming the buckling shape function as series of sinusoidal half waves in the 
direction of the axial compression. Timoshenko used the boundary conditions to set up a matrix which was solved to obtain the buckling loads. He also investigated and found solutions for the elastic stability problem of uniaxially compressed rectangular thin plates with two opposite edges simply supported and the other edges under various support conditions.

Considerable research works have been presented on the stability of plates of various shapes, types and boundary conditions. Some significant contributions to the research on plate stability are reported by: Gambhir [2], Bulson [3], Chajes [4], Timoshenko and Gere [5], Shi [8], Shi and Bezine [9], Ullah et al [10, 11, 12, 13], Wang et al [14], Abodi [15], Yu [16], Abolghasemi et al [17], Xiang et al [18] and Bouazza et al [19]

Contemporary research work on the plate stability problems have used various numerical methods such as the differential quadrature method (DQM), discrete singular convolution (DSC) method, harmonic differential quadrature method, ordinary finite difference method (FDM), meshfree method, generalized Galerkin method, finite strip method, B-spline finite strip method, exact finite strip method, hp-cloud method, modified Ishlinskii's solution method, meshless analog equation method, finite element method (FEM), extended Kantorovich method (EKM) and pb2-Ritz method. Very recent research work on the subject of plate stability using various numerical and analytical techniques have been reported by Lopatin and Morozov [20], Ghannadpour et al [21], Jafari and Azhari [22], Zureick [23], Seifi et al [24], Li et al [25], Wang et al [26], Mandal and Mishra [27], Shama [28], and Yao and Fujikubo [29].

Oguaghamba [30] studied the buckling and post buckling load characteristics of thin rectangular plates with isotropic, homogeneous material properties. Oguaghamba et al [31] studied the buckling and post buckling load characteristics of thin rectangular clamped plates made of isotropic, homogeneous materials. Ibearugbulem [32] used the Ritz direct variational method to study the elastic stability problems of thin rectangular flat plates under uniaxial uniform compressive load for various boundary conditions. Ibearugbulem et al [33] and Osadebe et al [34] used the Taylor-Maclaurin's series shape functions in the Galerkin's variational method for the elastic stability analysis of simply supported, isotropic, homogeneous thin rectangular plates under uniaxial uniform in-plane compressive load.

Elastic buckling problems of thin plates have been solved using Finite Difference method by Abodi [15]. Boundary element method has been applied to solve elastic buckling problem of orthotropic plates by Shi [8], and Shi and Bezine [9]. Nwoji et al [35] used the two-dimensional finite Fourier sine integral transformation method to obtain solutions for the elastic stability problem of Kirchhoff plates under uniaxial uniform compressive loads for the case of Dirichlet boundary conditions. They found that the two-dimensional finite Fourier sine integral transformation converts the domain PDE to an integral equation over the plate domain. They also found that the integral equation simplifies to an algebraic equation giving exact closed-form solutions for the buckling loads for all buckling modes. They found that the integral Kernel function in the transform adopted satisfies all the Dirichlet boundary conditions along all the edges for the simply supported plate studied.

Onah et al [36] solved the elastic buckling problem of thin rectangular plates with two opposite simply supported edges under uniform compressive loads and two clamped edges. They used the single finite Fourier sine integral transform method which they found suitable for the problem since the Dirichlet boundary conditions are identically satisfied by the integral kernel. They found that the domain equation is transformed to an integral equation, and ultimately to a system of ODEs. They solved the ODEs subject to the boundary conditions to set up a system of homogeneous algebraic equations in the integration constants. They used the conditions for nontrivial solutions to obtain the characteristic buckling equations whose eigenvalues were used to obtain the buckling loads. They obtained solutions that agreed with previous solutions in the literature.

Onyia et al [37] used the Galerkin-Kantorovich method for the elastic buckling analysis of rectangular thin SCSC plates. They found that the Galerkin-Kantorovich formulation of the elastic stability problem of thin plates is an integral equation that further simplified to a system of homogeneous fourth order ordinary differential equations (ODEs) in the unknown function. They obtained the general solution to the unknown function in terms of four sets of constants of integration and enforced boundary conditions to set up a system of homogeneous algebraic equations in terms of the integration constants. The condition for nontrivial solution was used to obtain the characteristic buckling equation as a transcendental equation which was solved using computer software based iteration methods. They obtained the buckling loads from the eigenvalues of the buckling equation, and found the solutions were exact for the buckling modes. They found that the characteristic buckling equation was identical to previously obtained equations.

In this study the single finite Fourier sine integral transform method is used to solve the elastic stability problem of rectangular thin SSCF plates under uniaxial uniform compressive loads on the simply supported edges.

\section{II.THEORETICAL FRAMEWORK}

The elastic buckling thin plate theory used is expressed in the rectangular Cartesian coordinates $x, y, z$ where $x$ and $y$ axis coincide with the middle plane and the $z$ coordinate axis is directed downwards from the middle plane as shown in Figure 1 .

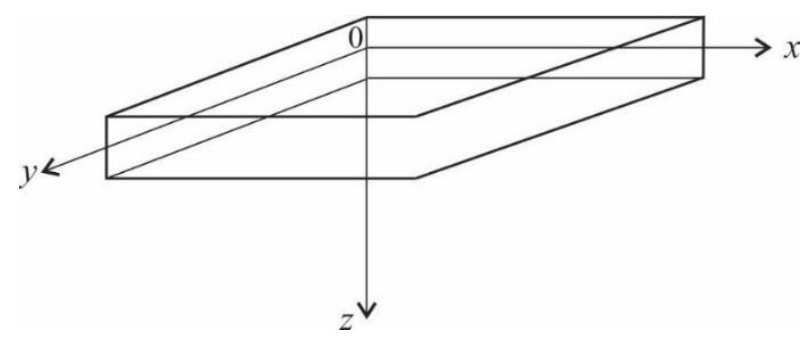

Fig. 1. Cartesian coordinate axes for the plate 
The origin of the Cartesian coordinates is defined at a corner of the plate as shown in Figure 1. A rectangular thin plate of inplane characteristic dimensions $a$ by $b$ and thickness $t$ is considered. The fundamental assumptions are:

(i) The deflections are very small as compared to the plate thickness.

(ii) The middle plane does not suffer deformation and is a neutral surface.

(iii) The plate thickness $t$ is small compared to the other inplane characteristic dimensions ( $a$ and $b$ ).

(iv) Plane cross-sections of the plate rotate during bending deformation, but remain perpendicular to the neutral plane; and do not distort. Hence the stresses and strains are proportional to their distance from the neutral surface.

The governing equation of elastic stability of plate is obtained by simultaneous consideration of the static equations of inplane equilibrium, transverse equilibrium and moment equilibrium. The equations are expressed in terms of stress resultants which are defined in terms of the stresses as follows:

$$
\begin{aligned}
& N_{x}=\int_{-t / 2}^{t / 2} \sigma_{x x} d z \\
& N_{y}=\int_{-t / 2}^{t / 2} \sigma_{y y} d z \\
& N_{x y}=N_{y x}=\int_{-t / 2}^{t / 2} \tau_{x y} d z \\
& M_{x}=\int_{-t / 2}^{t / 2} \sigma_{x x} z d z \\
& M_{y}=\int_{-t / 2}^{t / 2} \sigma_{y y} z d z \\
& M_{x y}=-M_{y x}=\int_{-t / 2}^{t / 2} \tau_{x y} z d z \\
& Q_{x}=\int_{-t / 2}^{t / 2} \tau_{x y} d z \\
& Q_{y}=\int_{-t / 2}^{t / 2} \tau_{y z} d z
\end{aligned}
$$

where $\sigma_{x x}, \sigma_{y y}, \sigma_{z z}$ are normal stresses $\tau_{x y}, \tau_{y z}, \tau_{x z}$ are shear stresses, $Q_{x}$ and $Q_{y}$ are shear forces, $N_{x}, N_{y}, N_{x y}, N_{y x}$ are the inplane forces, $M_{x}, M_{y}$ are bending moments and $M_{x y}, M_{y x}$ are twisting moments.

The equations of in-plane equilibrium are:

$$
\begin{aligned}
& \frac{\partial N_{x}}{\partial x}+\frac{\partial N_{y x}}{\partial y}=0 \\
& \frac{\partial N_{y}}{\partial y}+\frac{\partial N_{x y}}{\partial x}=0
\end{aligned}
$$

The equation of transverse equilibrium is:

$$
\frac{\partial Q_{x}}{\partial x}+\frac{\partial Q_{y}}{\partial y}+N_{x} \frac{\partial^{2} w}{\partial x^{2}}+N_{y} \frac{\partial^{2} w}{\partial y^{2}}+2 N_{x y} \frac{\partial^{2} w}{\partial x \partial y}=0
$$

The equations of moment equilibrium are:

$$
\begin{aligned}
& \frac{\partial M_{x y}}{\partial x}-\frac{\partial M_{y}}{\partial y}+Q_{y}=0 \\
& \frac{\partial M_{x}}{\partial x}-\frac{\partial M_{y x}}{\partial y}-Q_{x}=0
\end{aligned}
$$

Substitution of expressions for $Q_{x}$ and $Q_{y}$ obtained from Equation (4) into Equation (3), gives:

$$
\begin{aligned}
& \frac{\partial^{2} M_{x y}}{\partial y \partial x}-\frac{\partial^{2} M_{y}}{\partial y^{2}}+\frac{\partial Q_{y}}{\partial y}=0 \\
& \frac{\partial^{2} M_{x}}{\partial x^{2}}-\frac{\partial^{2} M_{y x}}{\partial x \partial y}-\frac{\partial Q_{x}}{\partial x}=0
\end{aligned}
$$

Substitution of expressions for $\frac{\partial Q_{x}}{\partial x}$ and $\frac{\partial Q_{y}}{\partial y}$ obtained from Equation (5) into Equation (3), gives the governing equation for elastic buckling as:

$$
\begin{aligned}
& \frac{\partial^{2} M_{x}}{\partial x^{2}}-2 \frac{\partial^{2} M_{x y}}{\partial x \partial y}+\frac{\partial^{2} M_{y}}{\partial y^{2}}+N_{x} \frac{\partial^{2} w}{\partial x^{2}}+ \\
& N_{y} \frac{\partial^{2} w}{\partial y^{2}}+2 N_{x y} \frac{\partial^{2} w}{\partial x \partial y}=0
\end{aligned}
$$

The moment deflection equations are used to express the governing equation in terms of transverse deflections, $w(x, y)$.

The stress-strain relations for the plane stress problem are given by:

$$
\begin{aligned}
& \sigma_{x}=\frac{E}{1-\mu^{2}}\left(\varepsilon_{x}+\mu \varepsilon_{y}\right) \\
& \sigma_{y}=\frac{E}{1-\mu^{2}}\left(\varepsilon_{y}+\mu \varepsilon_{x}\right) \\
& \tau_{x y}=G \gamma_{x y}=\frac{E}{2(1+\mu)} \gamma_{x y}
\end{aligned}
$$

where $\varepsilon_{x}, \varepsilon_{y}$ are normal strains, $\gamma_{x y}$ is the shear strain, $E$, is the Young's modulus of elasticity, $\mu$ is the Poisson's ratio, $G$ is the shear modulus or modulus of rigidity.

The displacement field components are:

$$
\begin{aligned}
& u=-z \frac{\partial w}{\partial x} \\
& v=-z \frac{\partial w}{\partial y}
\end{aligned}
$$


where $u$ is the displacement field component in the $x$ direction, $v$ is the displacement field component in the $y$ direction.

The kinematics relations are for linear infinitesimal elasticity problems, given by:

$\varepsilon_{x}=\frac{\partial u}{\partial x}$

$\varepsilon_{y}=\frac{\partial v}{\partial y}$

$\gamma_{x y}=\frac{\partial u}{\partial y}+\frac{\partial v}{\partial x}$

Thus, the strains are expressed in terms of the transverse deflection $w$ as:

$\varepsilon_{x}=-z \frac{\partial^{2} w}{\partial x^{2}}$

$\varepsilon_{y}=-z \frac{\partial^{2} w}{\partial y^{2}}$

$\gamma_{x y}=-2 z \frac{\partial^{2} w}{\partial x \partial y}$

The stress-strain relations are then:

$\sigma_{x}=\frac{-E z}{\left(1-\mu^{2}\right)}\left(\frac{\partial^{2} w}{\partial x^{2}}+\mu \frac{\partial^{2} w}{\partial y^{2}}\right)$

$\sigma_{y}=\frac{-E z}{\left(1-\mu^{2}\right)}\left(\frac{\partial^{2} w}{\partial y^{2}}+\mu \frac{\partial^{2} w}{\partial x^{2}}\right)$

$\tau_{x y}=\frac{-E z}{1+\mu} \frac{\partial^{2} w}{\partial x \partial y}$

The stress resultants are obtained from Equation (1) as:

$M_{x}=-D\left(\frac{\partial^{2} w}{\partial x^{2}}+\mu \frac{\partial^{2} w}{\partial y^{2}}\right)$

$M_{y}=-D\left(\frac{\partial^{2} w}{\partial y^{2}}+\mu \frac{\partial^{2} w}{\partial x^{2}}\right)$

$M_{x y}=-M_{y x}=-D(1-\mu) \frac{\partial^{2} w}{\partial x \partial y}$

where $D=\frac{E t^{3}}{12\left(1-\mu^{2}\right)}$

$D$ is the flexural rigidity of the plate.

Substitution of Equation (12) in Equation (6) gives the elastic buckling equation in terms of $w$ as:

$D\left(\frac{\partial^{4} w}{\partial x^{4}}+2 \frac{\partial^{4} w}{\partial x^{2} \partial y^{2}}+\frac{\partial^{4} w}{\partial y^{4}}\right)+N_{x} \frac{\partial^{2} w}{\partial x^{2}}+$

$$
N_{y} \frac{\partial^{2} w}{\partial y^{2}}+2 N_{x y} \frac{\partial^{2} w}{\partial x \partial y}=0
$$

Or

$D \nabla^{4} w+N_{x} \frac{\partial^{2} w}{\partial x^{2}}+N_{y} \frac{\partial^{2} w}{\partial y^{2}}+2 N_{x y} \frac{\partial^{2} w}{\partial x \partial y}=0$

where $\nabla^{4}=\frac{\partial^{4}}{\partial x^{4}}+2 \frac{\partial^{4}}{\partial x^{2} \partial y^{2}}+\frac{\partial^{4}}{\partial y^{4}}=\nabla^{2} \nabla^{2}$

$\nabla^{4}$ is the biharmonic operator, $\nabla^{2}$ is the Laplace operator.

\section{II.I The SSCF thin plate considered}

The SSCF thin plate considered, as shown in Figure 2 is simply supported along the edges $x=0$, and $x=a$, clamped along the edge $y=0$ and free along $y=b$.

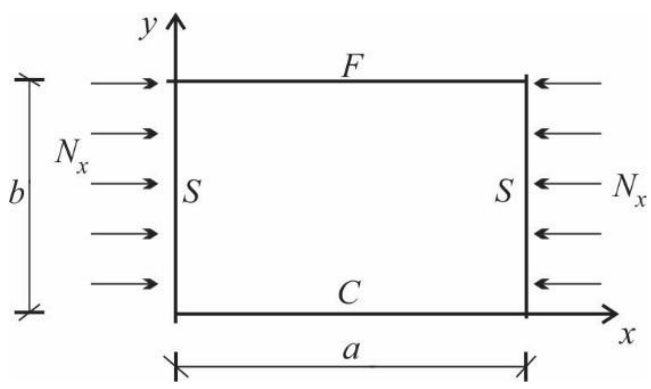

Fig. 2. SSCF thin plate studied

The SSCF plate is subject only to uniaxial uniform compressive load $N_{x}$ as shown in Figure 2. The governing domain equation to be solved is thus:

$D \nabla^{4} w+N_{x} \frac{\partial^{2} w}{\partial x^{2}}=0$

Since $N_{y}=0, N_{x y}=0$

The boundary conditions are:

at the simply supported edges $x=0, x=a$,

$w(x=0, y)=0$

$w(x=a, y)=0$

$M_{x}(x=0, y)=0 \Rightarrow \frac{\partial^{2} w}{\partial x^{2}}(x=0, y)=0$

$M_{x}(x=a, y)=0 \Rightarrow \frac{\partial^{2} w}{\partial x^{2}}(x=a, y)=0$

clamped edge $y=0$,

$w(x, y=0)=0$ 
$\frac{\partial w}{\partial y}(x, y=0)=0$

free edge $y=b$

$M_{y y}(x, y=b)=0$

$\left.V_{y z}\right|_{(x, y=b)}=-\left.D\left(\frac{\partial^{3} w}{\partial y^{3}}+(2-\mu) \frac{\partial^{3} w}{\partial x^{2} \partial y}\right)\right|_{(x, y=b)}=0$

$V_{y z}$ is the effective shear forces.

\section{METHODOLOGY}

The one dimensional finite Fourier sine integral transformation is applied to the domain PDE to obtain the integral equation:

$\int_{0}^{a}\left(\frac{\partial^{2} w}{\partial x^{4}}+2 \frac{\partial^{4} w}{\partial x^{2} \partial y^{2}}+\frac{\partial^{4} w}{\partial y^{4}}+\frac{N_{x}}{D} \frac{\partial^{2} w}{\partial x^{2}}\right) \sin \frac{n \pi x}{a} d x=0$

where the sinusoidal integral kernel function satisfies the boundary conditions at the simply supported edges, $x=0$, and $x=a$.

Using the linearity properties of the transformation, we obtain:

$\int_{0}^{a} \frac{\partial^{4} w(x, y)}{\partial x^{4}} \sin \frac{n \pi x}{a} d x+2 \frac{d^{2}}{d y^{2}} \int_{0}^{a} \frac{d^{2} w(x, y)}{d x^{2}} \sin \frac{n \pi x}{a} d x+$

$\frac{d^{4}}{d y^{4}} \int_{0}^{a} w(x, y) \sin \frac{n \pi x}{a} d x+\frac{N_{x}}{D} \int_{0}^{a} \frac{\partial^{2} w(x, y)}{\partial x^{2}} \sin \frac{n \pi x}{a} d x=0$

Due to the Dirichlet boundary conditions along the simply supported edges, $x=0$, and $x=a$,

$$
\begin{gathered}
\int_{0}^{a} \frac{\partial^{2} w(x, y)}{\partial x^{2}} \sin \frac{n \pi x}{a} d x=-\left(\frac{n \pi}{a}\right)^{2} \int_{0}^{a} w(x, y) \sin \frac{n \pi x}{a} d x \\
=-\left(\frac{n \pi}{a}\right)^{2} W(n, y)
\end{gathered}
$$

where $W(n, y)=\int_{0}^{a} w(x, y) \sin \frac{n \pi x}{a} d x$

$W(n, y)$ is the finite sine integral transform of $w(x, y)$. The inversion formula is the infinite series.

$$
\begin{gathered}
w(x, y)=\frac{2}{a} \sum_{n=1}^{\infty} W(n, y) \sin \frac{n \pi x}{a} \\
\int_{0}^{a} \frac{\partial^{4} w(x, y)}{\partial x^{4}} \sin \frac{n \pi x}{a} d x=-\left(\frac{n \pi}{a}\right)^{4} \int_{0}^{a} w(x, y) \sin \frac{n \pi x}{a} d x \\
=-\left(\frac{n \pi}{a}\right)^{4} W(n, y)
\end{gathered}
$$

Then, we obtain:

$$
\begin{gathered}
\left(\frac{n \pi}{a}\right)^{4} W(n, y)-2\left(\frac{n \pi}{a}\right)^{2} \frac{d^{2}}{d y^{2}} W(n, y)+\frac{d^{4}}{d y^{4}} W(n, y)- \\
\left(\frac{n \pi}{a}\right) \frac{N_{x}}{D} W(n, y)=0
\end{gathered}
$$

Simplifying,

$$
\begin{aligned}
& \frac{d^{4} W(n, y)}{d y^{4}}-2\left(\frac{n \pi}{a}\right)^{2} \frac{d^{2}}{d y^{2}} W(n, y)+ \\
& \left(\left(\frac{n \pi}{a}\right)^{4}-\left(\frac{n \pi}{a}\right)^{2} \frac{N_{x}}{D}\right) W(n, y)=0
\end{aligned}
$$

Or

$\frac{d^{4} W(n, y)}{d y^{4}}-2 \alpha_{n}^{2} \frac{d^{2} W(n, y)}{d y^{2}}+\left(\alpha_{n}^{4}-\alpha_{n}^{2} \frac{N_{x}}{D}\right) W(n, y)=0$

where $\alpha_{n}=n \pi / a$

\section{III.I General solution for the fourth order ordinary differential equation (ODE)}

By the method of trial functions, we assume a solution in the exponential form as:

$W(n, y)=A \exp \beta y$

where $\beta$ and $A$ are unknown parameters.

For nontrivial solutions, the characteristic or auxiliary equation is obtained as:

$$
\beta^{4}-2 \alpha_{n}^{2} \beta^{2}+\left(\alpha_{n}^{4}-\alpha_{n}^{2} \frac{N_{x}}{D}\right)=0
$$

Solving,

$$
\left(\beta^{2}-\alpha_{n}^{2}\right)^{2}-\alpha_{n}^{2} \frac{N_{x}}{D}
$$

Taking the square root of both sides of Equation (33), we have:

$$
\beta^{2}-\alpha_{n}^{2}=\sqrt{\alpha_{n}^{2} \frac{N_{x}}{D}}
$$

Solving for $\beta^{2}$, we have:

$$
\beta^{2}=\alpha_{n}^{2} \pm \sqrt{\alpha_{n}^{2} \frac{N_{x}}{D}}
$$

Hence, $\beta^{2}=\alpha_{n}^{2}+\sqrt{\alpha_{n}^{2} \frac{N_{x}}{D}}$

or $\beta^{2}=\alpha_{n}^{2}-\sqrt{\alpha_{n}^{2} \frac{N_{x}}{D}}=-\left(-\alpha_{n}^{2}+\left(\alpha_{n}^{2} \frac{N_{x}}{D}\right)^{1 / 2}\right)$ 
International Journal of Engineering Research and Technology. ISSN 0974-3154, Volume 13, Number 6 (2020), pp. 1147-1158

(C) International Research Publication House. https://dx.doi.org/10.37624/IJERT/13.6.2020.1147-1158

Hence, taking the square root of Equation (36) and (37), we have:

$\beta=\left(\alpha_{n}^{2}+\sqrt{\alpha_{n}^{2} \frac{N_{x}}{D}}\right)^{1 / 2}=\Omega_{1}$

$\beta= \pm i\left(\sqrt{\alpha_{n}^{2} \frac{N_{x}}{D}}-\alpha_{n}^{2}\right)= \pm i \Omega_{2}$

where $\Omega_{1}=\left(\sqrt{\alpha_{n}^{2} \frac{N_{x}}{D}}+\alpha_{n}^{2}\right)^{1 / 2}$

$\Omega_{2}=\left(\sqrt{\alpha_{n}^{2} \frac{N_{x}}{D}}-\alpha_{n}^{2}\right)^{1 / 2}$

The general solution is thus obtained from the basis of linearly independent solutions as:

$$
\begin{aligned}
W(n, y)=c_{1} \cosh \Omega_{1} y+c_{2} & \sinh \Omega_{1} y+ \\
& c_{3} \cos \Omega_{2} y+c_{4} \sin \Omega_{2} y
\end{aligned}
$$

where $c_{1}, c_{2}, c_{3}$ and $c_{4}$ are the four constants of integration.

\section{III.II Enforcement of boundary conditions}

Application of the finite Fourier sine transformation to the boundary conditions gives as follows:

$$
\begin{aligned}
& \int_{0}^{a} w(x, 0) \sin \frac{n \pi x}{a} d x=W(n, y=0)=0 \\
& \int_{0}^{a} \frac{\partial w(x, 0)}{\partial y} \sin \frac{n \pi x}{a} d x=\frac{d}{d y} \int_{0}^{a} w(x, 0) \sin \frac{n \pi x}{a} d x=0 \\
& \frac{d W}{d y}(x, y=0)=0
\end{aligned}
$$

$$
\begin{aligned}
& \int_{0}^{a} M_{y y}(x, y=b) \sin \frac{n \pi x}{a} d x= \\
& \int_{0}^{a}-D\left(\frac{\partial^{2} w}{\partial y^{2}}+\mu \frac{\partial^{2} w}{\partial x^{2}}\right) \sin \frac{n \pi x}{a} d x=0 \\
& -D\left\{\int_{0}^{a}\left(\frac{\partial^{2} w(x, b)}{\partial y^{2}}+\mu \frac{\partial^{2} w(x, b)}{\partial x^{2}}\right) \sin \frac{n \pi x}{a} d x\right\}=0 \\
& \frac{d^{2}}{d y^{2}} W(n, b)-\mu \alpha_{n}^{2} W(n, b)=0 \\
& \int_{0}^{a}-D\left(\frac{\partial^{3} w(x, b)}{\partial y^{3}}+(2-\mu) \frac{\partial^{3} w(x, b)}{\partial x^{2} \partial y}\right) \sin \frac{n \pi x}{a} d x=0 \\
& \frac{d^{3}}{d y^{3}} W(n, b)-(2-\mu) \alpha_{n}^{2} \frac{d W}{d y}(n, b)=0
\end{aligned}
$$

\section{RESULTS}

By differentiation with respect to $y$ :

$$
\begin{gathered}
W^{\prime}(n, y)=c_{1} \Omega_{1} \sinh \Omega_{1} y+c_{2} \Omega_{1} \cosh \Omega_{1} y- \\
c_{3} \Omega_{2} \sin \Omega_{2} y+c_{4} \Omega_{2} \cos \Omega_{2} y \\
W^{\prime \prime}(n, y)=c_{1} \Omega_{1}^{2} \cosh \Omega_{1} y+c_{2} \Omega_{1}^{2} \sinh \Omega_{1} y- \\
c_{3} \Omega_{2}^{2} \cos \Omega_{2} y-c_{4} \Omega_{2}^{2} \sin \Omega_{2} y \\
W^{\prime \prime \prime}(n, y)=c_{1} \Omega_{1}^{3} \sinh \Omega_{1} y+c_{2} \Omega_{1}^{3} \cosh \Omega_{1} y+ \\
c_{3} \Omega_{2}^{3} \sin \Omega_{2} y-c_{4} \Omega_{2}^{3} \cos \Omega_{2} y
\end{gathered}
$$

Hence using the equations, Equations (43, 45, 48 and 50), we obtain:

$$
W(n, y=0)=c_{1}+c_{3}=0
$$

Solving Equation (54) for $c_{3}$, we have:

$\therefore c_{3}=-c_{1}$

$W^{\prime}(n, y=0)=c_{2} \Omega_{1}+c_{4} \Omega_{2}=0$

Solving Equation (56) for $c_{4}$, we have:

$$
c_{4}=-\frac{c_{2} \Omega_{1}}{\Omega_{2}}
$$

The use of Equation (48) gives:

$$
\begin{array}{r}
c_{1} \Omega_{1}^{2} \cosh \Omega_{1} b+c_{2} \Omega_{1}^{2} \sinh \Omega_{1} b-c_{3} \Omega_{2}^{2} \cos \Omega_{2} b- \\
c_{4} \Omega_{2}^{2} \sin \Omega_{2} b-\mu \alpha_{n}^{2}\left(c_{1} \cosh \Omega_{1} b+c_{2} \sinh \Omega_{1} b+\right. \\
\left.c_{3} \cos \Omega_{2} b+c_{4} \sin \Omega_{2} b\right)=0
\end{array}
$$

Simplifying, Equation (58) give:

$$
\begin{aligned}
& c_{1}\left(\Omega_{1}^{2}-\mu \alpha_{n}^{2}\right) \cosh \Omega_{1} b+c_{2}\left(\Omega_{1}^{2}-\mu \alpha_{n}^{2}\right) \sinh \Omega_{1} b- \\
& c_{3}\left(\Omega_{2}^{2}+\mu \alpha_{n}^{2}\right) \cos \Omega_{2} b-c_{4}\left(\Omega_{2}^{2}+\mu \alpha_{n}^{2}\right) \sin \Omega_{2} b=0
\end{aligned}
$$

Hence, using Equations (55) and (57), we simplify Equation (55) as follows:

$$
\begin{aligned}
& c_{1}\left(\Omega_{1}^{2}-\mu \alpha_{n}^{2}\right) \cosh \Omega_{1} b+c_{2}\left(\Omega_{1}^{2}-\mu \alpha_{n}^{2}\right) \sinh \Omega_{1} b+ \\
& c_{1}\left(\Omega_{2}^{2}+\mu \alpha_{n}^{2}\right) \cos \Omega_{2} b+\frac{c_{2} \Omega_{1}}{\Omega_{2}}\left(\Omega_{2}^{2}+\mu \alpha_{n}^{2}\right) \sin \Omega_{2} b=0(60)
\end{aligned}
$$

Using Equation (50), we have:

$$
\begin{aligned}
& c_{1} \Omega_{1}^{3} \sinh \Omega_{1} b+c_{2} \Omega_{1}^{3} \cosh \Omega_{1} b+c_{3} \Omega_{2}^{3} \sin \Omega_{2} b- \\
& c_{4} \Omega_{2}^{3} \cos \Omega_{2} b-(2-\mu) \alpha_{n}^{2}\left(c_{1} \Omega_{1} \sinh \Omega_{1} b+c_{2} \Omega_{1} \cosh \Omega_{1} b-\right. \\
& \left.c_{3} \Omega_{2} \sin \Omega_{2} b+c_{4} \Omega_{2} \cos \Omega_{2} b\right)=0
\end{aligned}
$$

Simplifying Equation (61) we have:

$$
\begin{aligned}
& c_{1}\left(\Omega_{1}^{3}-(2-\mu) \alpha_{n}^{2} \Omega_{1}\right) \sinh \Omega_{1} b+c_{2}\left(\Omega_{1}^{3}-\right. \\
& \left.(2-\mu) \alpha_{n}^{2} \Omega_{1}\right) \cosh \Omega_{1} b+c_{3}\left(\Omega_{2}^{3}+(2-\mu) \alpha_{n}^{2} \Omega_{2}\right) \sin \Omega_{2} b- \\
& c_{4}\left(\Omega_{2}^{3}+(2-\mu) \alpha_{n}^{2} \Omega_{2}\right) \cos \Omega_{2} b=0
\end{aligned}
$$


International Journal of Engineering Research and Technology. ISSN 0974-3154, Volume 13, Number 6 (2020), pp. 1147-1158

(C) International Research Publication House. https://dx.doi.org/10.37624/IJERT/13.6.2020.1147-1158

Further simplification of Equation (62) gives:

$$
\begin{gathered}
c_{1} \Omega_{1}\left(\Omega_{1}^{2}-(2-\mu) \alpha_{n}^{2}\right) \sinh \Omega_{1} b+c_{2} \Omega_{1}\left(\Omega_{1}^{2}-\right. \\
\left.(2-\mu) \alpha_{n}^{2}\right) \cosh \Omega_{1} b+c_{3} \Omega_{2}\left(\Omega_{2}^{2}+(2-\mu) \alpha_{n}^{2}\right) \sin \Omega_{2} b- \\
c_{4} \Omega_{2}\left(\Omega_{2}^{2}+(2-\mu) \alpha_{n}^{2}\right) \cos \Omega_{2} b=0
\end{gathered}
$$

The boundary condition equations can be simplified using Equation (64) which can be proved.

$\Omega_{1}^{2}-\mu \alpha_{n}^{2}=\Omega_{2}^{2}+(2-\mu) \alpha_{n}^{2}$

From Equation (64), we have:

$\Omega_{2}^{2}-\Omega_{1}^{2}=-\mu \alpha_{n}^{2}-(2-\mu) \alpha_{n}^{2}=-\mu \alpha_{n}^{2}-2 \alpha_{n}^{2}+\mu \alpha_{n}^{2}=-2 \alpha_{n}^{2}$

From Equations (40) and (41) we have:

$$
\begin{aligned}
& \Omega_{2}^{2}-\Omega_{1}^{2}=\sqrt{\alpha_{n}^{2} \frac{N_{x}}{D}}-\alpha_{n}^{2}-\left(\sqrt{\alpha_{n}^{2} \frac{N_{x}}{D}}+\alpha_{n}^{2}\right)=-2 \alpha_{n}^{2}(66) \\
& \therefore \Omega_{2}^{2}-\Omega_{1}^{2}=-2 \alpha_{n}^{2}
\end{aligned}
$$

Thus, Equation (64) is proved. Let us define a new parameter $\lambda_{1}$ as follows:

$$
\Omega_{1}^{2}-\mu \alpha_{n}^{2}=\Omega_{2}^{2}+(2-\mu) \alpha_{n}^{2}=\lambda_{1}
$$

Similarly, the following Equation (68) can be proved where we define another new parameter $\lambda_{2}$ for simplification of the resulting expressions:

$\Omega_{2}^{2}+\mu \alpha_{n}^{2}=\Omega_{1}^{2}-(2-\mu) \alpha_{n}^{2}=\lambda_{2}$

From Equation (68) we have:

$\Omega_{2}^{2}-\Omega_{1}^{2}=-(2-\mu) \alpha_{n}^{2}-\mu \alpha_{n}^{2}=-2 \alpha_{n}^{2}+\mu \alpha_{n}^{2}-\mu \alpha_{n}^{2}=-2 \alpha_{n}^{2}$

In terms of the parameters, $\lambda_{1}$ and $\lambda_{2}$, the boundary conditions are expressed as:

$$
\begin{gathered}
c_{1}\left(\lambda_{1} \cosh \Omega_{1} b+\lambda_{2} \cos \Omega_{2} b\right)+ \\
c_{2}\left(\lambda_{1} \sinh \Omega_{1} b+\frac{\Omega_{1}}{\Omega_{2}} \lambda_{2} \sin \Omega_{2} b\right)=0 \\
c_{1}\left(\Omega_{1} \lambda_{2} \sinh \Omega_{1} b-\Omega_{2} \lambda_{1} \sin \Omega_{2} b\right)+ \\
c_{2}\left(\Omega_{1} \lambda_{2} \cosh \Omega_{1} b+\Omega_{1} \lambda_{1} \cos \Omega_{2} b\right)=0
\end{gathered}
$$

In matrix form, we have:

$$
\left(\begin{array}{cc}
\left(\lambda_{1} \cosh \Omega_{1} b+\lambda_{2} \cos \Omega_{2} b\right) & \left(\lambda_{1} \sinh \Omega_{1} b+\frac{\Omega_{1}}{\Omega_{2}} \lambda_{2} \sin \Omega_{2} b\right) \\
\left(\Omega_{1} \lambda_{2} \sinh \Omega_{1} b-\Omega_{2} \lambda_{1} \sin \Omega_{2} b\right) & \left(\Omega_{1} \lambda_{2} \cosh \Omega_{1} b+\Omega_{1} \lambda_{1} \cos \Omega_{2} b\right)
\end{array}\right)\left(\begin{array}{l}
c_{1} \\
c_{2}
\end{array}\right)=\left(\begin{array}{l}
0 \\
0
\end{array}\right)
$$

For nontrivial solutions $\left(\begin{array}{l}c_{1} \\ c_{2}\end{array}\right) \neq 0$
The elastic stability equation is expressed in determinant form as:

$\left|\begin{array}{cc}\left(\lambda_{1} \cosh \Omega_{1} b+\lambda_{2} \cos \Omega_{2} b\right) & \left(\lambda_{1} \sinh \Omega_{1} b+\frac{\Omega_{1}}{\Omega_{2}} \lambda_{2} \sin \Omega_{2} b\right. \\ \left(\Omega_{1} \lambda_{2} \sinh \Omega_{1} b-\Omega_{2} \lambda_{1} \sin \Omega_{2} b\right) & \left(\Omega_{1} \lambda_{2} \cosh \Omega_{1} b+\Omega_{1} \lambda_{1} \cos \Omega_{2} b\right)\end{array}\right|=0$

Expanding the determinant, the stability equation becomes:

$\Omega_{1} \lambda_{1} \lambda_{2} \cosh ^{2} \Omega_{1} b+\lambda_{2}^{2} \Omega_{1} \cosh \Omega_{1} b \cos \Omega_{2} b+$

$\lambda_{1}^{2} \Omega_{1} \cosh \Omega_{1} b \cos \Omega_{2} b+\lambda_{1} \lambda_{2} \Omega_{1} \cos ^{2} \Omega_{2} b-$

$\left(\Omega_{1} \lambda_{1} \lambda_{2} \sinh ^{2} \Omega_{1} b+\frac{\Omega_{1}}{\Omega_{2}} \lambda_{2} \Omega_{1} \lambda_{2} \sinh \Omega_{1} b \sin \Omega_{2} b-\right.$

$\left.\lambda_{1}^{2} \Omega_{2} \sinh \Omega_{1} b \sin \Omega_{2} b-\frac{\Omega_{1}^{2}}{\Omega_{2}} \lambda_{1} \lambda_{2} \Omega_{2} \sin ^{2} \Omega_{2} b\right)=0$

Simplifying, the elastic stability equation becomes:

$$
\begin{gathered}
\lambda_{1} \lambda_{2} \Omega_{1}\left(\cosh ^{2} \Omega_{1} b-\sinh ^{2} \Omega_{1} b\right)+\lambda_{1} \lambda_{2} \Omega_{1}\left(\cos ^{2} \Omega_{2} b+\right. \\
\left.\sin ^{2} \Omega_{2} b\right)+\left(\lambda_{2}^{2} \Omega_{1}+\lambda_{1}^{2} \Omega_{1}\right) \cosh \Omega_{1} b \cos \Omega_{2} b- \\
\left(\frac{\lambda_{2}^{2} \Omega_{1}^{2}}{\Omega_{2}}-\lambda_{1}^{2} \Omega_{2}\right) \sinh \Omega_{1} b \sin \Omega_{2} b=0
\end{gathered}
$$

We recall the following trigonometric and hyperbolic identities

$$
\begin{aligned}
& \cosh ^{2} \theta-\sinh ^{2} \theta=1 \\
& \cos ^{2} \theta+\sin ^{2} \theta=1
\end{aligned}
$$

From Equations (76) and (77) we note that:

$$
\begin{aligned}
& \cosh ^{2} \Omega_{1} b-\sinh ^{2} \Omega_{1} b=1 \\
& \cos ^{2} \Omega_{2} b+\sin ^{2} \Omega_{2} b=1
\end{aligned}
$$

Using Equations (78) and (79), the buckling equation Equation (75) - further simplifies as:

$$
\begin{array}{r}
2 \lambda_{1} \lambda_{2} \Omega_{1}+\left(\lambda_{2}^{2}+\lambda_{1}^{2}\right) \Omega_{1} \cosh \Omega_{1} b \cos \Omega_{2} b- \\
\left(\frac{\lambda_{2}^{2} \Omega_{1}^{2}}{\Omega_{2}}-\lambda_{1}^{2} \Omega_{2}\right) \sinh \Omega_{1} b \sin \Omega_{2} b=0
\end{array}
$$

Division of Equation ( 80$)$ by $\Omega_{1}$ gives:

$$
\begin{aligned}
2 \lambda_{1} \lambda_{2}+ & \left(\lambda_{2}^{2}+\lambda_{1}^{2}\right) \cosh \Omega_{1} b \cos \Omega_{2} b- \\
& \frac{1}{\Omega_{1}}\left(\frac{\lambda_{2}^{2} \Omega_{1}^{2}}{\Omega_{2}}-\lambda_{1}^{2} \Omega_{2}\right) \sinh \Omega_{1} b \sin \Omega_{2} b=0
\end{aligned}
$$

Thus, we obtain the elastic stability equation as the transcendental equation:

$$
\begin{aligned}
2 \lambda_{1} \lambda_{2}+ & \left(\lambda_{1}^{2}+\lambda_{2}^{2}\right) \cosh \Omega_{1} b \cos \Omega_{2} b- \\
& \frac{1}{\Omega_{1} \Omega_{2}}\left(\lambda_{2}^{2} \Omega_{1}^{2}-\lambda_{1}^{2} \Omega_{2}^{2}\right) \sinh \Omega_{1} b \sin \Omega_{2} b=0
\end{aligned}
$$


The transcendental equation is solved using computer based iteration software to obtain the eigenvalues from which the critical buckling loads are determined for various values of the plate aspect ratio and for Poisson's ratio $\mu=0.25$. The critical buckling load coefficients $K(a / b)$ of SSCF plates for $\mu=0.25$ and various values of the aspect ratio $r=a / b$ are presented in Table 1 for uniaxial uniform compressive load applied at the simply supported edges $x=0$, and $x=a$.

Table 1. Critical buckling load factors (coefficients) $K(a / b)$ for SSCF rectangular plates under uniform axial compression $N_{x_{c r}}=K(a / b) D \pi^{2} / b^{2}$

\begin{tabular}{|c|c|c|c|}
\hline$r=a / b$ & $\begin{array}{c}\text { Corresponding } \\
\text { buckling mode }\end{array}$ & $\begin{array}{c}\text { Present study } \\
K(a / b)\end{array}$ & $\begin{array}{c}\text { Wang et al [1] } \\
K(a / b)\end{array}$ \\
\hline 0.5 & $n=1$ & 4.518 & 4.518 \\
\hline 1.0 & $n=1$ & 1.698 & 1.698 \\
\hline 1.5 & $n=1$ & 1.339 & 1.339 \\
\hline 2.0 & $n=1$ & 1.386 & 1.386 \\
\hline 2.5 & $n=2$ & 1.432 & 1.432 \\
\hline 3.0 & $n=2$ & 1.339 & 1.339 \\
\hline 3.5 & $n=2$ & 1.336 & 1.336 \\
\hline 4.0 & $n=3$ & 1.386 & 1.386 \\
\hline 4.5 & $n=3$ & 1.339 & 1.339 \\
\hline 5.0 & $n=3$ & 1.329 & 1.329 \\
\hline 5.5 & $n=3$ & 1.347 & 1.347 \\
\hline 6.0 & $n=3$ & 1.339 & 1.339 \\
\hline
\end{tabular}

\section{IV.I Imposition of boundary conditions for SSSS Plates}

For SSSS plates, the boundary conditions along the $y=0$ and $y$ $=b$ edges are:

$w(x, y=0)=0$

$w(x, y=b)=0$

$\frac{\partial^{2} w}{\partial y^{2}}(x, y=0)=0$

$\frac{\partial^{2} w}{\partial y^{2}}(x, y=b)=0$

Application of the finite Fourier sine integral transform to the boundary conditions gives:

$\int_{0}^{a} w(x, y=0) \sin \frac{n \pi x}{a} d x=W(n, y=0)=0$

$$
\begin{gathered}
\int_{0}^{a} w(x, y=b) \sin \frac{n \pi x}{a} d x=W(n, y=b)=0 \\
\int_{0}^{a} \frac{\partial^{2} w}{\partial y^{2}}(x, y=0) \sin \frac{n \pi x}{a} d x=\frac{\partial^{2}}{\partial y^{2}} \int_{0}^{a} w(x, y=0) \sin \frac{n \pi x}{a} d x \\
=\frac{d^{2}}{d y^{2}} W(n, y=0)=W^{\prime \prime}(n, y=0)=0
\end{gathered}
$$

$$
\begin{gathered}
\int_{0}^{a} \frac{\partial^{2} w}{\partial y^{2}}(x, y=b) \sin \frac{n \pi x}{a} d x=\frac{\partial^{2}}{\partial y^{2}} \int_{0}^{a} w(x, y=b) \sin \frac{n \pi x}{a} d x \\
=\frac{d^{2}}{d y^{2}} W(n, y=b)=W^{\prime \prime}(n, y=b)=0
\end{gathered}
$$

Hence,

$$
\begin{aligned}
& W(n, y=0)=c_{1}+c_{3}=0 \\
& W^{\prime \prime}(n, y=0)=c_{1} \Omega_{1}^{2}-c_{3} \Omega_{2}^{2}=0 \\
& W(n, y=b)=c_{1} \cosh \Omega_{1} b+c_{2} \sinh \Omega_{1} b+ \\
& c_{3} \cos \Omega_{2} b+c_{4} \sin \Omega_{2} b=0 \\
& W^{\prime \prime}(n, y=b)=c_{1} \Omega_{1}^{2} \cosh \Omega_{1} b+c_{2} \Omega_{1}^{2} \sinh \Omega_{1} b- \\
& c_{3} \Omega_{2}^{2} \cos \Omega_{2} b-c_{4} \Omega_{2}^{2} \sin \Omega_{2} b=0
\end{aligned}
$$

Solving, from Equation (88),

$c_{1}=-c_{3}$

Then substitution into Equation (89) gives

$c_{1} \Omega_{1}^{2}+c_{1} \Omega_{2}^{2}=c_{1}\left(\Omega_{1}^{2}+\Omega_{2}^{2}\right)=0$

Since $\Omega_{1}^{2}+\Omega_{2}^{2} \neq 0$, then

$c_{1}=0$

$c_{3}=0$

The system of homogeneous equations then simplifies to:

$$
\begin{aligned}
& c_{2} \sinh \Omega_{1} b+c_{4} \sin \Omega_{2} b=0 \\
& c_{2} \Omega_{1}^{2} \sinh \Omega_{1} b-c_{4} \Omega_{2}^{2} \sin \Omega_{2} b=0
\end{aligned}
$$

In matrix form, we have

$$
\left(\begin{array}{cc}
\sinh \Omega_{1} b & \sin \Omega_{2} b \\
\Omega_{1}^{2} \sinh \Omega_{1} b & -\Omega_{2}^{2} \sin \Omega_{2} b
\end{array}\right)\left(\begin{array}{l}
c_{2} \\
c_{4}
\end{array}\right)=\left(\begin{array}{l}
0 \\
0
\end{array}\right)
$$

The characteristic stability equation is found from the condition for nontrivial solutions which is the vanishing of the determinant of the coefficient matrix. Thus

$\left|\begin{array}{cc}\sinh \Omega_{1} b & \sin \Omega_{2} b \\ \Omega_{1}^{2} \sinh \Omega_{1} b & -\Omega_{2} \sin \Omega_{2} b\end{array}\right|=0$

Expansion of the determinant gives:

$-\Omega_{2}^{2} \sin \Omega_{2} b \sinh \Omega_{1} b-\Omega_{1}^{2} \sinh \Omega_{1} b \sin \Omega_{2} b=0$ 
International Journal of Engineering Research and Technology. ISSN 0974-3154, Volume 13, Number 6 (2020), pp. 1147-1158

(C) International Research Publication House. https://dx.doi.org/10.37624/IJERT/13.6.2020.1147-1158

Simplifying, Equation (100) becomes:

$\sin \Omega_{2} b \sinh \Omega_{1} b\left(-\Omega_{1}^{2}-\Omega_{2}^{2}\right)=0$

For nontrivial solutions, the characteristic buckling equation has infinite number of roots (eigenvalues) found by solving:

$\sin \Omega_{2} b=0$

Or $\sinh \Omega_{1} b=0$

Solving,

$\Omega_{2} b=\sin ^{-1} 0=m \pi, \quad m=1,2,3,4, \ldots$

$\therefore \Omega_{2}=\frac{m \pi}{b}$

Then using Equation (41) we have:

$\Omega_{2}=\left(\sqrt{\frac{\alpha_{n}^{2} N_{x}}{D}}-\alpha_{n}^{2}\right)^{1 / 2}=\frac{m \pi}{b}$

Squaring both sides of Equation (106), we have:

$\sqrt{\frac{\alpha_{n}^{2} N_{x}}{D}}-\alpha_{n}^{2}=\left(\frac{m \pi}{b}\right)^{2}$

Hence,

$\sqrt{\frac{\alpha_{n}^{2} N_{x}}{D}}=\alpha_{n}^{2}+\left(\frac{m \pi}{b}\right)^{2}=\left(\frac{n \pi}{a}\right)^{2}+\left(\frac{m \pi}{b}\right)^{2}$

Squaring both sides of Equation (108), we obtain:

$\frac{\alpha_{n}^{2} N_{x}}{D}=\left(\left(\frac{n \pi}{a}\right)^{2}+\left(\frac{m \pi}{b}\right)^{2}\right)^{2}$

Simplifying,

$$
N_{x}=\frac{D}{\alpha_{n}^{2}}\left(\left(\frac{n \pi}{a}\right)^{2}+\left(\frac{m \pi}{b}\right)^{2}\right)^{2}
$$

Hence,

$N_{x}=D\left(\frac{a}{n \pi}\right)^{2}\left(\left(\frac{n \pi}{a}\right)^{2}+\left(\frac{m \pi}{b}\right)^{2}\right)^{2}$

Simplifying,

$N_{x}=\frac{D a^{2} \pi^{4}}{n^{2} \pi^{2}}\left(\left(\frac{n}{a}\right)^{2}+\left(\frac{m}{b}\right)^{2}\right)^{2}=D \pi^{2} \frac{a^{2}}{n^{2}}\left(\frac{n^{2}}{a^{2}}+\frac{m^{2}}{b^{2}}\right)^{2}$

Simplifying further,

$N_{x}=D \pi^{2} \frac{a^{2}}{n^{2}}\left(\frac{n^{4}}{a^{4}}+\frac{2 n^{2} m^{2}}{a^{2} b^{2}}+\frac{m^{4}}{b^{4}}\right)$
Hence,

$$
N_{x}=D \pi^{2}\left(\frac{n^{2}}{a^{2}}+\frac{2 m^{2}}{b^{2}}+\frac{m^{4} a^{2}}{n^{2} b^{4}}\right)
$$

Let $a=b r$

$$
\begin{array}{r}
N_{x}=D \pi^{2}\left(\frac{n^{2}}{b^{2} r^{2}}+\frac{2 m^{2}}{b^{2}}+\frac{m^{4} b^{2} r^{2}}{n^{2} b^{4}}\right) \\
=\frac{D \pi^{2}}{b^{2}}\left(\frac{n^{2}}{r^{2}}+2 m^{2}+\frac{m^{4} r^{2}}{n^{2}}\right)
\end{array}
$$

Thus,

$$
N_{x}=K(r) \frac{D \pi^{2}}{b^{2}}=\left(\frac{n^{2}}{r^{2}}+2 m^{2}+\frac{m^{4} r^{2}}{n^{2}}\right) \frac{D \pi^{2}}{b^{2}}
$$

In which,

$$
K(r)=K(a / b)=\left(\frac{n^{2}}{r^{2}}+2 m^{2}+\frac{m^{4} r^{2}}{n^{2}}\right)
$$

For $m=1, n=1$,

$$
K(a / b)=K(r)=\left(\frac{1}{r^{2}}+2+r^{2}\right)=K_{x_{c r}}(r)
$$

$K(a / b)$ is calculated for varying values or $r$ where $0<r<1$ and presented in Table 2. Table 2 also displays results obtained previously for the critical elastic buckling load by Iyengar [7] and Nwoji et al [35] using a double finite Fourier sine integral transform method.

Table 2. Critical elastic buckling load factors (coefficients) for simply supported rectangular thin plates subjected to uniaxial uniform compressive load acting at $x=0$, and $x=a$ for various plate aspect ratios.

\begin{tabular}{|c|c|c|c|}
\hline $\begin{array}{c}\text { Plate aspect } \\
\text { ratio }(a / b)\end{array}$ & $\begin{array}{c}\text { Present study } \\
K(a / b)\end{array}$ & $\begin{array}{c}\text { Iyengar [7] } \\
K(a / b)\end{array}$ & $\begin{array}{c}\text { Nwoji et al [35] } \\
K(a / b)\end{array}$ \\
\hline 0.1 & 102.01 & 102.01 & 102.01 \\
\hline 0.2 & 27.04 & 27.04 & 27.04 \\
\hline 0.3 & 13.201111 & 13.2011 & 13.2011 \\
\hline 0.4 & 8.41 & 8.41 & 8.41 \\
\hline 0.5 & 6.25 & 6.25 & 6.25 \\
\hline 0.6 & 5.137778 & 5.1378 & 5.1378 \\
\hline 0.7 & 4.530816 & 4.5308 & 4.5308 \\
\hline 0.8 & 4.2025 & 4.2025 & 4.2025 \\
\hline 0.9 & 4.044568 & 4.0446 & 4.0446 \\
\hline 1.0 & 4 & 4 & 4 \\
\hline
\end{tabular}


International Journal of Engineering Research and Technology. ISSN 0974-3154, Volume 13, Number 6 (2020), pp. 1147-1158

(C) International Research Publication House. https://dx.doi.org/10.37624/IJERT/13.6.2020.1147-1158

\section{DISCUSSION}

This article has illustrated the application of the single finite Fourier sine integral transform method to the elastic stability problem of rectangular thin SSCF and SSSS plates. The problem is governed by BVP given for SSCF plates as Equations (17), (18), (19) and (20), where Equation (17) is the domain equation and Equations (18), (19) and (20) are the boundary conditions. The application of the one-dimensional finite sine integral transform to the governing domain equation converted the problem to an integral equation expressed as Equation (21). The sinusoidal kernel function of the transformation is found to satisfy all the Dirichlet boundary conditions along the simply supported edges $x=0$ and $x=a$. The integral equation is simplified using its linearity properties, integration by parts, and the Dirichlet conditions at $x=0$, and $x$ $=a$ to yield the system of homogeneous fourth order ODEs expressed as Equation (28). The general solution of the system of homogeneous ODEs is obtained as Equation (42) in terms of four sets of integration constants.

SSCF plates: Enforcement of the boundary conditions along the clamped and free edges gave a system of four homogeneous equations in terms of four sets of integration constants Equations (54), (56), (59) and (63). Equations (55) and (57) are used to further simplify the system of equations to a system of two homogeneous equations (70) and (71), which are expressed in matrix form as Equation (72). The condition for nontrivial solution gave the characteristic buckling equation as Equation (73) - a determinantal equation. The determinant was expanded and simplified to yield after using trigonometric and hyperbolic identities, the characteristic buckling equation as the transcendental equation - Equation (82). The eigenvalues of the transcendental equations obtained by iteration methods for various aspect ratios were used to find the critical buckling load factors (coefficients) $K(a / b)$. The critical buckling load coefficient calculated for various aspect ratios $(a / b)$ are presented in Table 1, as well as solutions previously obtained by Wang et al [1]. Table 1 shows that the solutions obtained in this study using the one-dimensional (1D) finite Fourier sine integral transform method are identical with results reported by Wang et al [1].

SSSS plates: The one-dimensional finite sine integral transformed boundary condition equations are found as Equations (84), (85), (86) and (87). Explicitly, the transformed boundary conditions are found in terms of the integration constants as Equations (88), (89), (90) and (91). Solutions of Equations (88) and (89) give Equations (94) and (95) and the equations reduce to Equations (96) and (97) which contain only two constants $c_{2}$ and $c_{4}$. The system of homogeneous equations then become in matrix form Equation (98). The characteristic stability equation, obtained from the condition for nontrivial solutions which is the vanishing of the determinant of the coefficient matrix is found as Equation (99). Expansion of the determinant and simplification of the resulting equation gave the stability equation as Equation (101). The solution is found by solving Equation (102) and is obtained as Equation (105). The eigenvalues of the equation are used to obtain the buckling load expression as Equation (116). The buckling load expression is written in terms of elastic buckling load coefficients $K(a / b)$ which depend upon the aspect ratio as
Equation (117). Critical elastic buckling load coefficients are found for $m=n=1$ as Equation (119) which is evaluated for varying values of the aspect ratio and presented in Table 2. Table 2 reveals that the obtained results of the present study are identical with solutions obtained by Iyengar [7] and Nwoji et al [35] in which Nwoji et al [35] used a double finite Fourier sine integral transform method.

\section{CONCLUSION}

In conclusion,

(i) The single finite Fourier sine integral transform method has been successfully used to solve the elastic stability problem of SSCF and SSSS rectangular thin plates subjected to uniaxial uniform compressive load along the simply supported edges $x=0$, and $x=a$.

(ii) The integral transform method used has proved ideally fitting for the problem because the integral kernel function of the transform is a sinusoidal function of the $x$ coordinate and satisfies all the Dirichlet boundary conditions along the simply supported edges.

(iii) The one-dimensional finite Fourier sine integral transform of the governing domain equation converts the boundary value problem (BVP) to an integral equation.

(iv) The resulting integral equation upon the use of linearity properties of the transformation and integration by parts, simplifies to a system of homogeneous ordinary differential equations (ODEs) in the unknown function, which is the single finite Fourier sine transform of $w(x, y)$.

(v) The system of ODEs is solved using the method of trial functions to obtain the solution basis for linearly independent solutions and then the general solution which has four sets of integration constants.

(vi) The employment of the boundary conditions along the edges $y=0$ and $y=b$ is used to set up a system of homogeneous equations in terms of the integration constants.

(vii) The requirement for nontrivial solutions of the homogeneous equations is used to find the characteristic elastic stability equation in determinantal form.

(viii) Ultimately, upon expansion of the determinant, the characteristic elastic stability equation is obtained as a transcendental equation which is solved using iteration methods and software tools to obtain the eigenvalues used to find the buckling loads.

(ix) The expressions obtained for the characteristic elastic buckling equation for the SSCF and SSSS plates are exact and identical with previously obtained expressions thus validating the study results.

(x) The elastic buckling loads obtained for both SSCF and SSSS plates are also exact since the governing equations were solved for all points in the solution domain (plate region) and all boundary conditions are also satisfied by the solution. 
(xi) The exact buckling loads for the SSCF and SSSS plates are identical with previously obtained values by other researchers.

\section{REFERENCES}

[1] Wang CM, Wang CY, Reddy JN., Exact solutions for buckling of structural members, Boca Raton Florida, CRC Press LLC, 2005.

[2] Gambhir ML., Elastic buckling of thin plates. In: Stability Analysis and Design of Structures, Springer Berlin Heidelberg, 2013. https://doi.org/10.1007/978-3-66209996-4_8.

[3] Bulson PS., The stability of flat plates, Chatto and Windus London, UK, 1970.

[4] Chajes A., Principles of structural stability theory, Englewood Cliffs New Jersey Prentice Hall, 1993.

[5] Timoshenko SP, Gere JM., Theory of elastic stability, McGraw Hill, New York, 1985.

[6] Timoshenko S., Theory of elastic stability, McGraw Hill Publishing Co Ltd, 1936. DOI: https://doi.org/10.1007/S0368393100104389.

[7] Iyengar NG., Structural stability of columns and plates, Chichester: Ellis Horwood, 1988.

[8] Shi G., Flexural vibration and buckling analysis of orthotropic plates by the boundary element method, International Journal of Solids and Structures, 1990; 26:1351-1370.

[9] Shi G, Bezine GP., Buckling analysis of orthotropic plates by boundary element method, Mechanics Research Communications, 1990; 17:1-8.

[10] Ullah S, Zhong Y, Zhang J., Analytical buckling solutions of rectangular thin plates by straightforward generalized integral transform method, International Journal of Mechanical Sciences, 2019; 152(2019): 535-544.

[11] Ullah S, Zhou J, Zhang J, Zhou C, Wang H, Zhong Y, Wang B, Li R., New analytic shear buckling solution of clamped rectangular plates by a two-dimensional generalized finite integral transform method, International Journal of Structural Stability and Dynamics, 2020; 20(2), 2017002 (10pages), DOI: $10.1142 / \mathrm{S} 0219455420710029$.

[12] Ullah S, Wang H, Zheng X, Zhang J, Zhong Y, Li R., New analytic buckling solutions of moderately thick clamped rectangular plates by a straightforward finite integral transform method, Archive of Applied Mechanics, September 2019; 89(9): 1885-1897.

[13] Ullah S, Zhang J, Zhong U., New analytic solutions of buckling problem of rotationally restrained rectangular thin plates, International Journal of Applied Mechanics, 2019; 11(10) 1950101 DOI: $10.1142 / \mathrm{S} 1758825119501011$
[14] Wang CM, Xiang Y, Chakrabarty J., Elastic/plastic buckling of thick plates, International Journal of Solids and Structures, 2001; 38: 8617-8640.

[15] Abodi JT., Elastic buckling analysis of plates under inplane patch loading using finite difference method, Journal of Kerbala University, 2012; 10(2): 142-150.

[16] Yu Chen., Buckling of rectangular plates under intermediate and end loads, MSc Thesis, Department of Civil Engineering, National University of Singapore, 2003.

[17] Abolghasemi S, Eipakchi H, Shariati M., An analytical solution for buckling of plates with circular cutout subjected to non-uniform in-plane loading, Archive of Applied Mechanics, 2019: 89: 2519-2543. https://doi.org/10.1007/S00419019-01592-3.

[18] Xiang Y, Wang CM, Wang CY., Buckling of rectangular plates with an internal hinge, International Journal of Structural Stability and Dynamics, 2001; 1(2): 169-179.

[19] Bouazza M, Quinas D, Yazid A, Hamouine A., Buckling of thin plates under uniaxial and biaxial compression, Journal of Material Science and Engineering, 2012: 32(8): 487-492.

[20] Lopatin AV, Morozov EV., Approximate buckling analysis of the CCFF orthotropic plates subjected to inplane loading, International Journal of Mechanical Sciences, 2014; 84(2014): 38-44.

[21] Ghannadpour S, Ovesy H, Zia-Dehkordi E., Buckling and post-buckling behaviour of moderately thick plates using an exact finite strip, Computers and Structures, 2015; 147(2015): 172-180.

[22] Jafari N, Azhari M., Buckling of moderately thick arbitrarily shaped plates with intermediate point supports using a simple hp-cloud method, Applied Mathematics and Computation, 2017; 313(2017): 196-208.

[23] Zureick AH., On the buckling of an isotropic rectangular plate uniformly compressed on two simply supported edges and with two free unloaded edges, Thin-walled Structures, 2018: 124(2018): 180-183.

[24] Seifi R, Chahardoli S, Attav AA., Axial buckling of perforated plates reinforced with strips and middle tubes, Mechanics Research Communications, 2017; 85(2017): 21-32.

[25] Li R, Zhang X, Wang H, Xiong S, Yan K, Li P., New analytic buckling solutions of rectangular thin plates with all edges free, International Journal of Mechanical Sciences, 2018; 144(2018): 67-73.

[26] Wang B, Li P, Li R., Symplectic superposition method for new analytic buckling solutions of rectangular thin plates, International Journal of Mechanical Sciences, 2016; 119(2016): 432-441.

[27] Mandal SK, Mishra PK., Buckling analysis of rectangular plate element subjected to in-plane loading using finite 
element method, International Journal of Scientific and Engineering Research, 2018; 9(4): 70-79.

[28] Shama M., Buckling of stiffened panels, Springer Berlin Heidelberg, 2013; 267-304.

[29] Yao T, Fujikubo M., "Chapter 5 - buckling/plastic collapse behaviour and strength of rectangular plates subjected to combined loads," in Buckling and Ultimate Strength of Ship and Ship-like Floating Structures, 2016; 157-206, Butterworth-Heinemann.

[30] Oguaghamba OA., Analysis of buckling and post buckling loads of isotropic thin rectangular plates, $\mathrm{PhD}$ Thesis, Post Graduate School, Federal University of Technology, Owerri, Nigeria, 2015.

[31] Oguaghamba OA, Ezeh JC, Ibearugbulem MO, Ettu LO., Buckling and post buckling loads characteristics of all edges clamped thin rectangular plates, The International Journal of Science, 2015; 4(11): 55-61.

[32] Ibearugbulem OA., Application of a direct variational principle in elastic stability analysis of thin rectangular flat plates, PhD Thesis, School of Post Graduate Studies, Federal University of Technology, Owerri, Nigeria, 2012.

[33] Ibearugbulem OM, Osadebe NN, Ezeh JC, Onwuka DO., Buckling analysis of axially compressed SSSS thin rectangular plate using Taylor-Maclaurin shape function, International Journal of Civil and Structural Engineering, 2011; 2(2): 676-681.

[34] Osadebe NN, Nwokike VC, Oguaghamba OA., Stability analysis of SSSS thin rectangular plate using multidegrees of freedom Taylor-Maclaurin's series in Galerkin variational method, Nigerian Journal of Technology, 2016; 35(3): 503-509.

[35] Nwoji CU, Onah HN, Mama BO, Ike CC, Ikwueze EU., Elastic buckling analysis of simply supported thin plates using the double finite Fourier sine integral transform method, Explorematics Journal of Innovative Engineering and Technology, 2017; 1(1): 37-47.

[36] Onah HN, Nwoji CU, Ike CC, Mama BO., Elastic buckling analysis of uniaxially compressed CCSS Kirchhoff plate using single finite Fourier sine integral transform method, Modelling, Measurement and Control B, 2018; 87(2): 107-111. DOI: 10.18280/mmc_b.870208.

[37] Onyia ME, Rowland-Lato EO, Ike CC., GalerkinKantorovich method for the elastic buckling analysis of thin rectangular SCSC plates, International Journal of Engineering Research and Technology, 2020; 13(4): 613619. 